\title{
Circuits in random graphs: from local trees to global loops
}

\author{
Enzo Marinari†, Rémi Monasson $\ddagger$ \\ $\dagger$ Dipartimento di Fisica, SMC-INFM and INFN, Università di Roma "La Sapienza", \\ P. A. Moro 2, 00185 Roma, Italy \\ $\ddagger$ CNRS-Laboratoire de Physique Théorique de l'ENS, 24 rue Lhomond, 75005 Paris, \\ France
}

\begin{abstract}
We compute the number of circuits and of loops with multiple crossings in random regular graphs. We discuss the importance of this issue for the validity of the cavity approach. On the one side we obtain analytic results for the infinite volume limit in agreement with existing exact results. On the other side we implement a counting algorithm, enumerate circuits at finite $N$ and draw some general conclusions about the finite $N$ behavior of the circuits.

PACS numbers: $02.10 . \mathrm{Ox}, 89.75 . \mathrm{Hc}, 05.40 .-\mathrm{a}$
\end{abstract}

\section{Introduction}

The study of random graphs, initiated more than four decades ago, has been since an issue of major interest in probability theory and in statistical mechanics. Examples of random graphs abound [1, from the original Erdös-Renyi model [2], where edges are chosen independently of each other between pairs of a set of $N$ vertices (with a fixed probability of $O(1 / N)$ ), to the scale-free graphs with power law degree distribution [3], only introduced in recent times. An interesting and useful distribution is the one that generates random $c$-regular graphs [4. These are uniformly drawn from the set of all graphs over $N$ vertices, each restricted to have degree $c$. Random regular graphs can be easily generated when $N$ is large (and $c$ finite) [5], and an instance of 3-regular graph is symbolized in figure 1. Around a randomly picked up vertex called root the graph looks like a regular tree. The probability that there exists a circuit (a self-avoiding closed path) of length $L$ going through the root vanishes when $N$ is sent to infinity and $L$ is kept finiteł [4].

The purpose of this article is to reach some quantitative understanding of the presence of long circuits in random graphs. Our motivation is at least two-fold.

First, improving our knowledge on circuits in random graphs would certainly have positive fall-out on the understanding of equilibrium properties of models of interacting $\ddagger$ More precisely, this probability asymptotically departs from zero when $\log N=O(L)$. Finite-length loops may be present in the graph, but not in extensive number. 


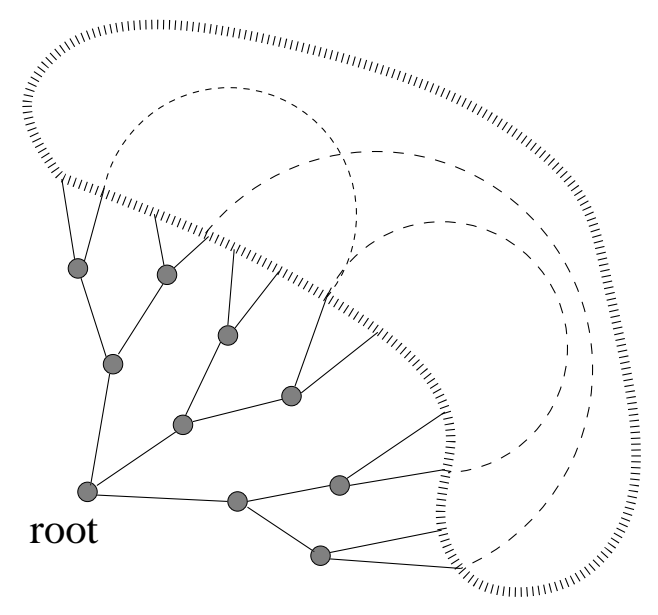

Figure 1. Structure of a random regular graph with degree $c=3$. In the vicinity of a vertex (the root) the graph looks like a (regular) tree attached to the bulk. For any integer $D$, vertices at distance $\leq D$ from the root are almost surely distinct when the size $N$ of the graph goes to infinity. No short loop, but many long (dashed) ones pass through the root.

variables living on these graphs. For instance, frustration in spin systems emerge from the presence of circuits with odd length. The number of these circuits is therefore directly related to the amount of glassiness present in the system at low temperature. From the dynamical point of view too, the properties of models of interacting or communicating agents e.g. routers, ... strongly depend on the topological properties of the underlying graph, and particularly upon feedbacks resulting from the presence of circuits. These "practical" incentives, together with purely academic motivations have led to recent studies on the distributions of unicyclic components [6] and cycles [7] in random graphs.

Secondly, the absence of short circuits in random graphs or, equivalently, the observation that random graphs essentially exhibit a local tree-like structure is crucial to the analytical treatment of spin models on random graphs with some common techniques, e.g. the replica or cavity methods. These models are characterized by (realistic) strong interactions between spins, in contrast to usual mean-field systems, e.g. Curie-Weiss or Sherrington-Kirkpatrick models. Yet, the absence of short-ranged loops in random graphs make them, around any vertex (root), locally similar to a tree surrounded by a remote bulk. Because of that one conjectures that the freeenergy of a spin model on a random graph is equal to the one on the tree with selfconsistent boundary conditions requiring that appropriate properties (for example the magnetization for the Ising model) are identical on the leaves and at the root vertex [8, 9, 10]. One of the goals of this note is to point out that this procedure $\grave{a}$ la Bethe amounts to make assumptions on the large-scale structure of random graphs, i.e. on the distribution of long loops§.

$\S$ This point has certainly already come to mind to researchers in the field but it is, at the best of our knowledge, never explicitly mentioned in the literature. 
We will first discuss loops, and compute their number in the thermodynamic limit by analyzing the partition function of the Ising model. Next we will compute the number of circuits by discussing the $n \longrightarrow 0$ limit of the $O(n)$ ferromagnetic model. For gathering information about the finite $N$ behavior of the number of loops (or of the loop entropy) we will use an effective counting algorithm. We will end this note with a discussion of our findings and of the (many) open issues.

\section{Infinite size limit: mean-field approach}

In this section, we enumerate long loops in random graphs from the study of associated statistical mechanics models. We will give our ad hoc definition of a loop in the following section.

\subsection{Loops with multiple crossings}

Let us consider for example Ising spins $S_{i}= \pm 1$ on vertices $i=1, \ldots, N$ of the graph of figure 1, and ferromagnetic couplings $J_{i j}=1$ on edges $(i, j)$. To calculate the partition function $Z(\beta)$ of the Ising model at inverse temperature $\beta$ on the graph of figure 1, we consider the leaves of the uncovered local tree i.e. vertices at distance $D$ from the root (in figure 1 the maximum drawn distance is $D=2$ ) [11, 8, 9, 12, 10, 13. At sufficiently large $\beta$, a spontaneous, say, positive magnetization $m$ is expected to be present in the bulk. As a result, the spins attached to the leaves will feel an external field $H>0$. Integrating these spins out will in turn produce an external field $H^{\prime}$ acting on spins at distance $D-1$ from the root, with [1]

$$
H^{\prime}=\frac{(c-1)}{\beta} \tanh ^{-1}[\tanh (\beta) \tanh (\beta H)] .
$$

After repeated iterations of this procedure, the field at the root reaches a stationary value, $H^{*}$, from which we can derive the magnetization $m=\tanh \left[\beta c H^{*}\right]$ and the freeenergy density

$$
f(\beta)=-\frac{c}{2 \beta} \ln 2\left(e^{-\beta}+e^{\beta} \cosh 2 \beta H^{*}\right)+\frac{c-1}{\beta} \ln 2 \cosh \left(\frac{\beta c H^{*}}{c-1}\right) .
$$

The critical inverse temperature is the smallest value of $\beta$ for which $H^{*}$ is non zero i.e. $\beta_{c}=\tanh ^{-1}\left(\frac{1}{c-1}\right)$.

As is well known, the high temperature expansion of the partition function $Z$ of the Ising model can be written as a sum over closed paths going through each vertex an even number of times and each edge at most once [14. Each path is given a weight $(\tanh \beta)^{L}$ depending upon its length $L$. In the following we will refer to such paths as loops. Gathering all loops with the same weight i.e. same length, we obtain

$$
Z(\beta)=2^{N}(\cosh \beta)^{\frac{c N}{2}} \sum_{L} M(L)(\tanh \beta)^{L},
$$


A

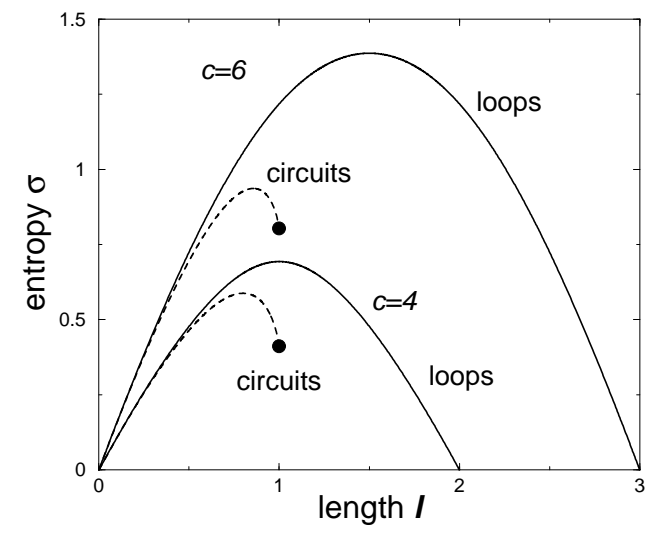

B

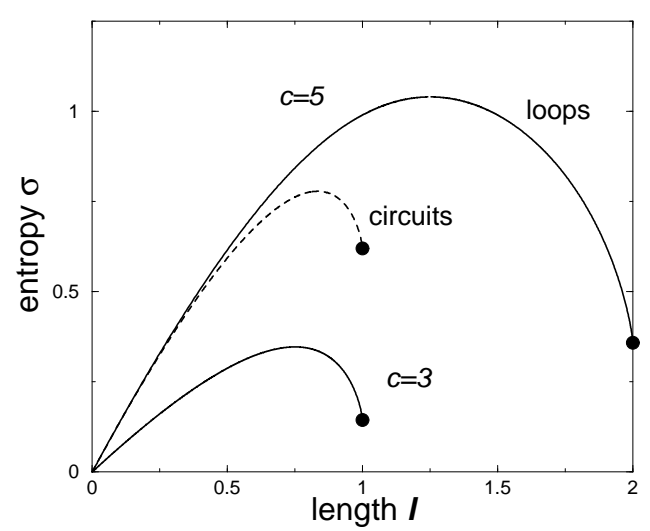

Figure 2. Entropy $\sigma$ as a function of the length $\ell$ of loops (full curves) and circuits (dashed curves) for even (A) and odd (B) degrees $c$ (for $c=3$, loops and circuits coincide). The slope at origin is $\ln (c-1)$ for both cases, the smallest loops being essentially circuits. Black dots, with coordinates $\ell_{+}, \sigma_{+}$identify the longest loops and circuits, see text.

where $M(L)$ is the number of loops of length $L$ that can be drawn on the graph. It is a sensible assumption that this multiplicity exhibits an exponential growth with the graph size,

$$
M(L=\ell N)=\exp [N \sigma(\ell)+o(N)],
$$

where $\ell$ is the intensive length of the loops, and $\sigma$ is the entropy of loops having length $\ell$. We stress here that $\ell=O(1)$ corresponds to $L=O(N)$. Knowledge of the entropy $\sigma$ will provide us with information about large-scale loops i.e. with lengths of the order of $N$. Insertion of the above scaling hypothesis for $M(L)$ in the partition function $Z$, and use of the Laplace method yield

$$
-\beta f(\beta)=-\ln 2-\frac{c}{2} \ln \cosh \beta+\max _{\ell}[\sigma(\ell)+\ell \ln \tanh \beta]
$$

in the infinite $N$ limit\|. In other words, the free-energy density $f(\beta)$ is essentially the Legendre transform of the loop entropy $\sigma(\ell)$.

We show in figure 2 our results for the loop entropy. The entropy departs from $\ell=\sigma=0$ with a slope equal to $-\ln \left(\tanh \beta_{c}\right)=\ln (c-1)$ from (15) 9 . Its maximum is reached in $\ell_{M}=\frac{c}{4}, \sigma_{M}=\left(\frac{c}{2}-1\right) \ln 2$, that is, for loops going through half of the edges. The left part of the entropy curve $\left(\ell \leq \ell_{M}\right)$ is parametrized by inverse temperatures $\beta$ going from $\beta_{c}(\ell=0$ - the para/ferro transition takes place when extensive loops start contributing to partition function $Z$ ) to $\infty$ (top of the curve - at zero temperature, $Z$ is dominated by the most numerous paths). The right part of the entropy curve $\left(\ell \geq \ell_{M}\right)$

$\|$ Here we are assuming that not only the free energy density $f$ is self-averaging (as is known and true) but that also $\sigma$ enjoys the same property.

I Note that the finite slope at origin comes from the fact that the objects counted here (loops or circuits) are non local; one cannot gain entropy by rearranging edges independently [15]. 
is obtained when $\tanh \beta>1$, that is, for inverse temperatures with an imaginary part equal to $\frac{\pi}{2}$ allowing to transform the hyperbolic tangent of $\beta$ into the cotangent of its real part. This is done by considering a real $\beta>\beta_{c}$, the stationary field $H^{*}$ for this temperature, and the transformation $\beta \rightarrow \beta+i \frac{\pi}{2},(\beta H)^{*} \rightarrow(\beta H)^{*}+i(c-1) \frac{\pi}{2}$.

The case of even degree. For even $c \sigma$ is left unchanged under the transformation $\ell \rightarrow \frac{c}{2}-\ell$. The right part of the entropy curve is thus the mirror symmetric of the left part with respect to $\ell=\ell_{M}$ (figure 2 $2 \mathrm{~A}$ ). This results from a duality between long and short extensive loops. Let $\Gamma^{F}$ be the full loop (all edges occupied, which is allowed for even $c$ ). Then, for any loop $\Gamma$ with length $\ell<\frac{c}{4}, \Gamma^{F} \backslash \Gamma$ (that is $\Gamma^{F}$ without the edges belonging to $\Gamma$ ) is a loop with length $\frac{c}{2}-\ell$. Notice that the largest loop is $\Gamma^{F}$, with length $\frac{c}{2}$.

The case of odd degree. Here duality does not hold and there is no simple transformation rule for $\ell$ and $\sigma$. An explicit calculation gives the entropy curve shown in figure 2 $2 \mathrm{~B}$. The maximal length $\ell_{+}=\frac{(c-1)}{2}$ is reached with an infinite slope, and corresponds to a finite entropy $\sigma_{+}=\frac{1}{2}((c-1) \ln (c-1)-(c-2) \ln c)$. For odd degrees $c$, indeed, loops cannot occupy all edges since the number of incoming edges onto each vertex is even. The longest loops have one free edge per vertex: these act as defects, the positions of which can be chosen with some freedom, giving rise to a finite entropy. The larger is $c$, the smaller is the ratio $\frac{\sigma_{+}}{\sigma_{M}}$ : intuitively, the frustration coming from the parity of $c$ is less and less important as $c$ increases ${ }^{+}$.

\subsection{Loops without crossings: circuits}

The iteration procedure can be applied to calculate the free-energy of other short-range models and then derive the entropies corresponding to large-scale diagrams generated through high temperature expansions. For instance the ferromagnetic $O(n)$ model with $n \rightarrow 0$ gives information on circuits i.e loops with vertices having degree 2 at most [16]. A spin $\vec{S}$ is submitted to two fields $H_{1}, H_{2}$ conjugated to the magnetization $\frac{\vec{S} \cdot \overrightarrow{1}}{\sqrt{n}}$ and its squared value*. The iteration equations for these fields read

$$
\begin{aligned}
H_{1}^{\prime} & =\frac{(c-1) \beta H_{1}}{1+H_{2}}, \\
H_{2}^{\prime} & =\frac{(c-1)(c-2) \beta^{2} H_{1}^{2}}{2\left(1+H_{2}\right)^{2}} .
\end{aligned}
$$

Inserting the fixed point values for the fields in the expression of the free-energy per spin component,

$$
f=\frac{1}{\beta} \ln \left[\left(1+H_{2}\right)^{c}\left(1+\frac{c(c-1) \beta^{2} H_{1}^{2}}{2\left(1+H_{2}\right)^{2}}\right)\right]-\frac{c}{2 \beta} \ln \left[\left(1+H_{2}\right)^{2}+\beta H_{1}^{2}\right],
$$

+ Numerical investigations of the one-step replica symmetry broken Ansatz for the Ising model with $\tanh \beta>1$ indicate that the replica symmetric solution is correct in this range of (complex-valued) temperature [15.

* Higher moments are not relevant in the $n \rightarrow 0$ limit. 
yields (for an alternative derivation, see [17]),

$$
f=-\frac{c-2}{2 \beta} \ln \left[\frac{c(c-1) \beta-2}{c-2}\right]+\frac{c}{2 \beta} \ln [(c-1) \beta] .
$$

The entropy of circuits,

$$
\sigma(\ell)=-(1-\ell) \ln (1-\ell)+\left(\frac{c}{2}-\ell\right) \ln \left(1-\frac{2 \ell}{c}\right)+\ell \ln (c-1),
$$

is obtained through $-\beta f(\beta)=\max _{\ell}[\sigma(\ell)+\ell \ln \beta]$. Results are shown in figure 2] The maximal entropy, $\sigma_{M}=\frac{1}{2}(c \ln (c-1)-(c-2) \ln (c+1))$, is reached in $\ell_{M}=\frac{c}{(c+1)}$. The rightmost point, with coordinates $\ell_{+}=1, \sigma_{+}=\ln (c-1)+\frac{1}{2}(c-2) \ln (1-2 / c)$ corresponds to Hamiltonian cycles. Expression (91) coincides with the output of rigorous calculations [2, 18, which shows the exactness of the replica symmetric hypothesis for the $O(n \rightarrow 0)$ model [17. Note the similarity of the entropy curves for loops on odd $c$-regular graphs and circuits (figure 2). In the two cases the slopes at minimal and maximal lengths are respectively finite and infinite, see the discussion above.

Let $c \geq 3$ and $M(L)$ be in all the rest of this note the number of circuits of length $L$ in a random, $c$-regular graph. For finite $L, M(L)$ is asymptotically Poisson-distributed when $N \rightarrow \infty$ [4],

$$
\operatorname{Proba}[M(L)=M]=\frac{1}{M !}\left[\frac{(c-1)^{L}}{2 L}\right]^{M} \exp \left\{-\frac{(c-1)^{L}}{2 L}\right\} .
$$

The above identity holds indeed for circuit-length $L \ll \log N$. The expected number of circuits of intensive length $\ell=L / N$ is therefore, for $\ell<\frac{\log N}{N}$,

$$
\langle M(\ell)\rangle=\frac{(c-1)^{L}}{2 L}=\exp \{N \sigma(\ell)-\log (N)+\tilde{\sigma}(\ell)\},
$$

with $\sigma(\ell)=\ell \log (c-1)$ and $\tilde{\sigma}(\ell)=\log (2 \ell)$. Note that this value of $\sigma(\ell)$ coincides with expression (9) at the first order in $\ell$.

\section{Finite size corrections to circuit number: numerical results}

To study what happens at finite $N$ we have implemented a fast algorithm for finding all circuits in a given graph [19]. It is important that we explicitly find, not only count, all the circuits so that by our method we can in principle give all interesting characterizations.

In our enumeration we first generate a random graph and then count the circuits. We average this outcome over a number of samples. We describe the algorithm for enumeration in next section.

To generate a fixed connectivity random graph we start by assuming that each site has $c$ connections that connect it to $c$ different sites: we allow no self-connections and no double edges. At the beginning, all connections are free. Two of the connections are extracted and matched together. We continue filling them up (we use a table that we resize at each step to keep the process effective) till all connections are set or we 
are stuck because there are two left connections that both belong to the same site or that belong to two sites that are already connected. To be sure not to introduce any systematic bias in this case we just discard the full graph and restart the procedure from scratch.

\subsection{An algorithm for circuit enumeration}

For enumerating circuits we have implemented and used an algorithm introduced by Johnson [19]. The algorithm finds all elementary circuits of a graph. The computer time needed for this task is bounded by $O((N+E)(M+1))$, where $N$ is the number of vertices of the graph, $E$ the number of edges and $M$ the total number of circuits in the graph: indeed one proves that the time used between the output of two consecutive circuits is bound by $O(N+E)$ (and that this is true also for time elapse before the output of the first circuit and after the output of the last one). The memory needed by the algorithm is bounded by $O(N+E)$.

Let us just sketch the crucial steps of the procedure. One first orders the vertices in some lexicographic sequence, and labels them with integers ranging from 0 to $N-1$. The search is started from a root vertex $r$, in the subgraph induced by $r$ and by vertices after $r$ : one of the crucial performance issues is that all vertices that become roots are the smallest vertex in at least one elementary circuit. The input to the procedure is the adjacency structure $A(v)$ for each vertex $v$, that represents the graph: it contains $u$ if and only if $(v, u) \in \mathcal{E}$, where $\mathcal{E}$ is the set of edges of the graph.

We block a vertex $v$ when it is added to a path beginning in $r$. We build elementary paths starting from $r$. The vertices of the current trial paths are loaded on a stack. A procedure adds the vertex to the path, if appropriate, and appends the vertex to the stack: the vertex is deleted from the stack when returning from this procedure. The ingenious part of the algorithm is in keeping a vertex blocked as long as possible, to minimize the execution time. This has to be done while keeping the procedure correct: the basic rule that has to be satisfied to guarantee that all circuits are found (only once) is that if it exists a path from the vertex $v$ to $r$ that does not intersect the path loaded on the stack, then $v$ has to be free (i.e. it cannot be in a blocked state). One uses a list to keep the information needed to satisfy this constraint while staying time effective.

Some details about performances are as follows: on an Intel Xeon $2.8 \mathrm{GHz}$ processor our implementation takes of the order of 0.07 seconds for finding all circuits of a $N=30$ graph (they are $O(50000)), 2.4 s$ for $N=40\left(O\left(1.510^{6}\right)\right.$ circuits) and $80 s$ for $N=50$ (with $O\left(410^{7}\right)$ circuits).

In order to test the results of our code we have also implemented a "quick and dirty" backtracking procedure to count Hamiltonian circuits. Since our procedure crucially depends on the quality of the random number generator we have also checked that different (high quality) random number generators lead to statistically compatible answers. 


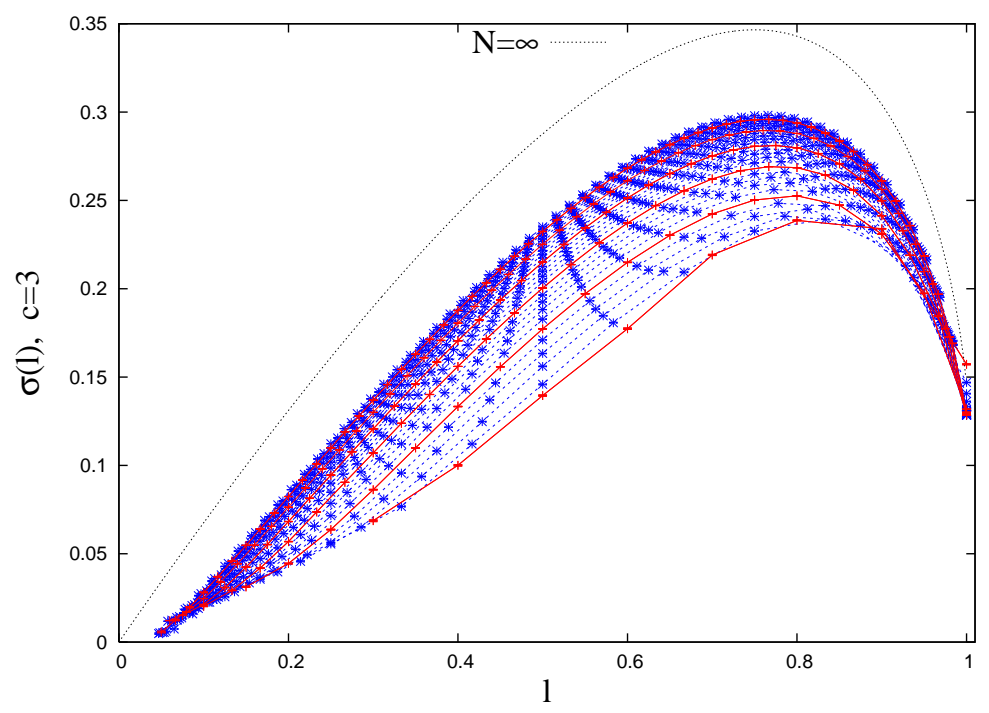

Figure 3. Circuit entropy $\sigma_{N}(\ell)$ as a function of $\ell$ for $c=3$, and for graph sizes ranging from $N=10$ to 64 (from bottom to top). The full curve corresponds to the analytical calculation of equation (9). Data for sizes multiple of 10 are made visible by using a different drawing style.

\subsection{Results and interpretation}

Thanks to our algorithm and implementation we have been able to enumerate of the order of $10^{14}$ circuits (a large number).

For small $c$ values we can study larger graphs (we have analyzed graphs with up to 64 vertices in the $c=3$ case and up to 22 vertices for $c=6$, and averaged our results over samples ranging from 1000 to 10000 random graphs). Typically we find for example of the order of 300 million circuits on a $N=56, c=3$ graph, one billion circuits on a $N=26, c=5$ graph and 1.5 billion circuits on a $N=22, c=6$ graph. For each value of $N$, we average over of the order of 10000 samples for all the $c=3$ enumerations, and 1000 graphs for $c>3$.

In figure 3 we plot the theoretical results obtained in the (replica symmetric) calculation of Section 2.2 for $c=3$, together with results obtained by finite enumeration on finite lattices with $N$ vertices. Numerical data from finite graphs are very slowly approaching the theoretical, infinite volume data. We try in the following to analyze the quality of this asymptotic agreement of the two sets of data.

At first we know that $\log \langle M(\ell)\rangle \sim \ell \log (c-1)$ as $\ell \rightarrow 0$ where $\langle M(\ell)\rangle$ is the average number of circuits of length $L=\ell \cdot N$. With our numerical data we cannot work really close to $\ell=0$, since for finite $N$ we have a value $\ell_{\min }$ that goes to zero as $N \rightarrow \infty$ : finite size effects appear as a flattening of $\log \langle M(\ell)\rangle$ when $\ell$ becomes very close to the minimum allowed value (see figure 3). One can easily see by eye that the slope is very similar to the asymptotic slope in the small $\ell$ region where we are relatively safe from finite size effects. We have fitted a linear behavior (that is indeed clear in the data) for example for $\ell$ in the range $(.13, .19)$ for $c=3$. 


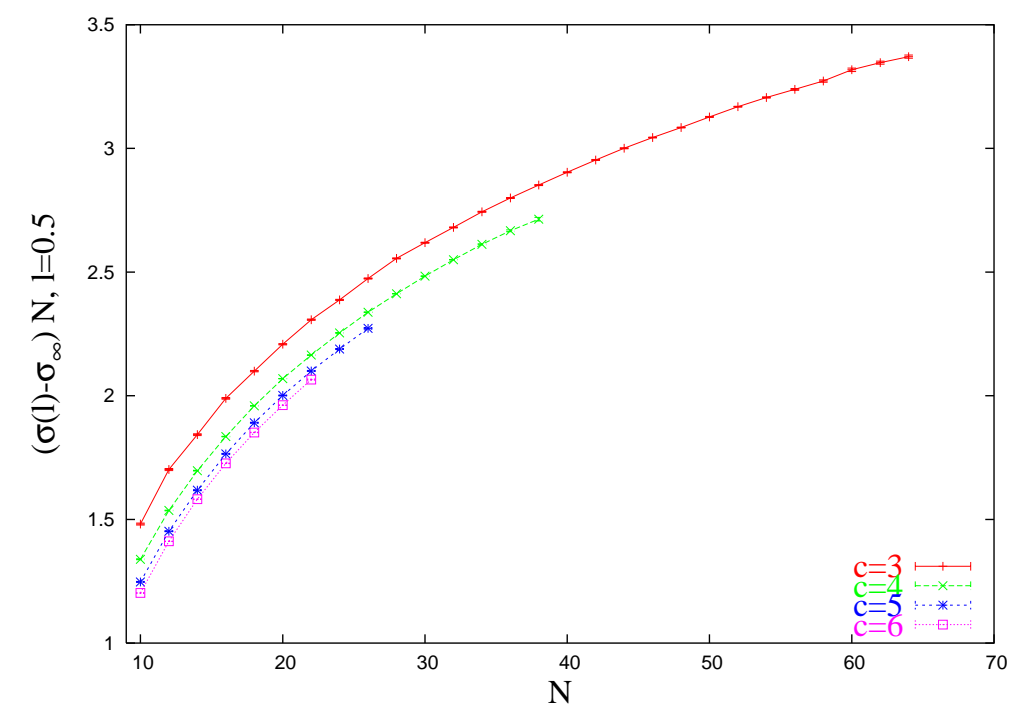

Figure 4. $N$ times the difference between the circuit entropy and its asymptotic value, as a function of $N$ for different connectivities $c$. Here $\ell=0.5$.

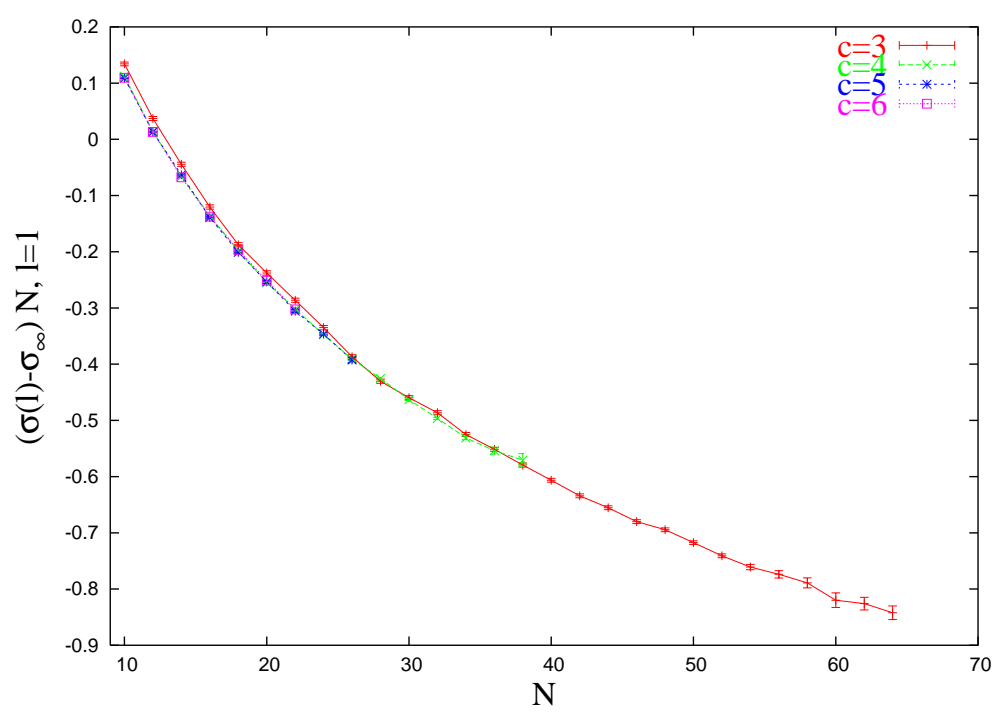

Figure 5. Same as in figure 4 but for Hamiltonian circuits i.e. $\ell=1$.

Using this approach we find for $c$ from 3 to 6 slopes about $20 \%$ smaller than the theoretical prediction (on the larger graphs we can study): for $c=3$ we find 0.54 versus a theoretical $\log 2 \sim 0.69$; for $c=4$ we find 0.87 versus 1.10 ; for $c=5$ we find 1.12 versus 1.39; for $c=6$ we find 1.31 versus 1.61. Finite size effect can be drastically reduced if we compare directly different $c$ value (since we are using graphs of a size that is limited by the same criterion for each $c$ value). For example the ratio of the slope of $c$ and $c+1$ is 0.62 for $c=3$ versus a theoretical $0.63,0.78$ versus 0.79 for $c=4$ and 0.85 versus 0.86 for $c=5$. This really remarkable agreement gives us confidence that we have a good control over finite size effects. 


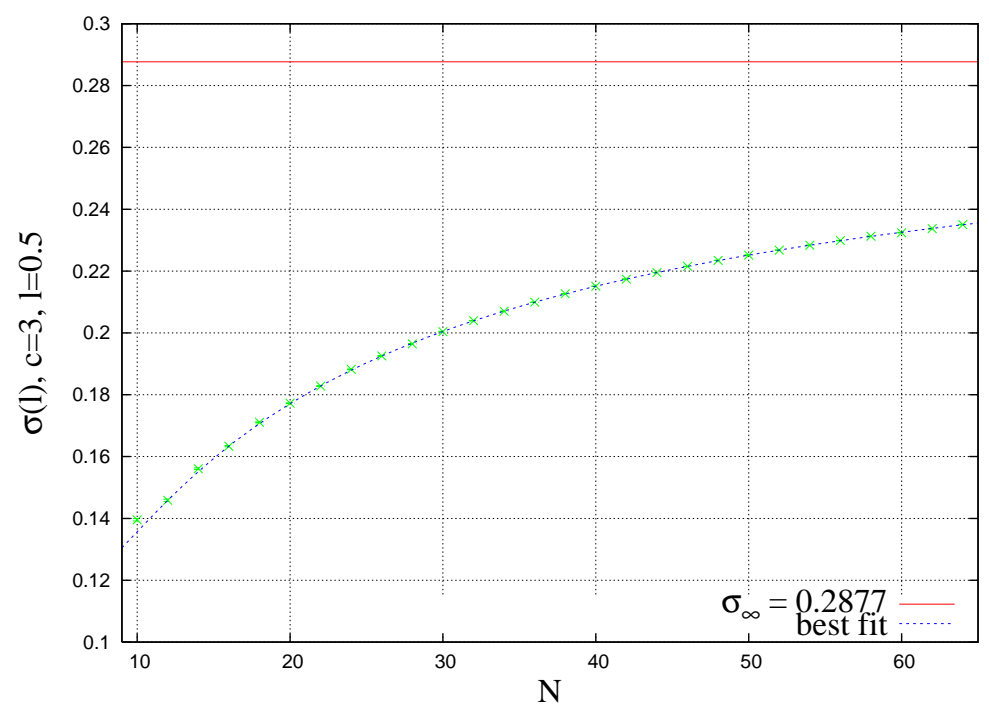

Figure 6. Circuit entropy $\sigma(\ell=0.5)$ versus $N$ for $c=3$ and best fit to the form (13).

Equation (111) tells us that the difference between the measured circuit entropy $\sigma_{N}(\ell)$ and the asymptotic value given by formula (9), once multiplied by $N$, behaves as

$$
\left(\sigma_{N}(\ell)-\sigma_{\infty}(\ell)\right) N=-\log N+\tilde{\sigma}(\ell)
$$

for vanishing small values of $\ell$, with $\tilde{\sigma}(\ell)=-\log (2 \ell)$. It is therefore independent of $c$, with a logarithmic dependence upon the graph size $N$. To check how equation (12) applies to finite values of $\ell$, we look first in figure 4 at the number of circuits with $\ell=0.5$, for different values of $N$ and $c$ (we have harvested our most precise data at $c=3$ ). Data indeed show only a very weak dependence upon $c$, and this dependence becomes weaker with increasing $c$. Data for $c=5$ are already indistinguishable from the ones for $c=6$.

In figure 5. we show the same quantity for $\ell=1$ i.e. for Hamiltonian circuits, that pass through all vertices of the random graph. As far as the scaling with $c$ is concerned figure 5 shows that the scaling of Hamiltonian circuits is excellent already at $c=3$. We will come back later about the fact that scaling property of Hamiltonian circuits turn out to be very different from the ones of all other finite $\ell$, less dense circuits.

Inspired by the result obtained for small circuits (11), (12) we look for the following fit for the circuit entropy for finite values of $\ell$,

$$
\sigma_{N}(l)=\sigma_{\infty}(l)+c_{1} \frac{\log N}{N}+c_{2} \frac{1}{N} .
$$

In figure 6 we show our results for $c=3, \ell=0.5$. The quality of the best fit to data with sizes $N \geq 30$ only is excellent, and is very good agreement with all data with $N \geq 12$. The two parameter fit is clearly superior to power law fits. We find that with very good accuracy (surely better than one percent)

$$
c_{1}=-1
$$




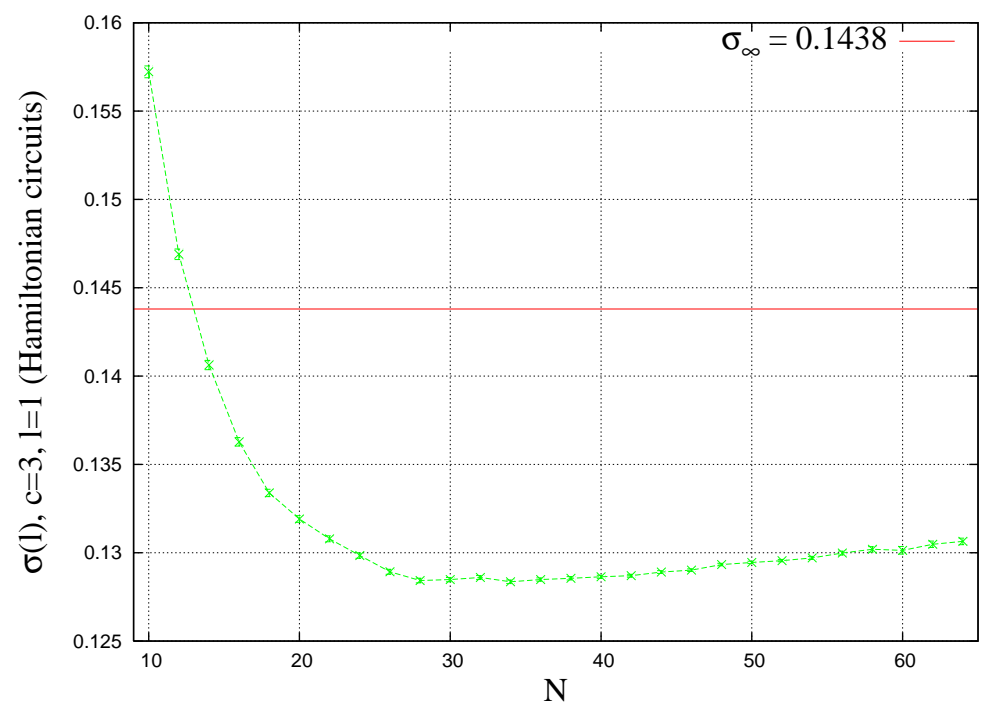

Figure 7. $\sigma(l=1)$ versus $N$ for $c=3$.

i.e. that even at finite $\ell$ the relation (12) gives the correct leading corrections. This result tells us that for all $\ell$ values (maybe excluding $\ell=1$, see later) we have that the average number of circuits of reduced length $\ell$ equals

$$
\langle M(\ell N)\rangle=(K(\ell)+o(1)) \frac{e^{N \sigma_{\infty}(\ell)}}{N},
$$

where $K(\ell)$ is a bounded function of $\ell$. For $c_{2}$, we find values close to 1 e.g. .78 in the case of $\ell=0.5$. Here precision is not as good since this is a sub-leading correction. What is clear from our data is that sub-leading corrections to the circuit entropy are of the order of $1 / N$ as encoded in formula (14).

As we have said above the case of Hamiltonian circuits $(\ell=1)$ is exceptional. Finite size transient effects are very strong; this is intuitively expected since these circuits fill the graph and are deeply affected by its finite size. We show in figure 7 the analogous of figure [ but for $\ell=1$. It is clear that the structure of finite size effects is completely different. On the contrary we have already explained that we find exactly the same behavior for all intermediate $\ell$ values: the case $\ell=1$ appears as isolated.

\section{Conclusions}

To sum up, the absence of small loops in random graphs allows one to argue that the free-energy of a spin model defined on the graph is equal to the one on a regular tree with a self-consistent external field at boundary (leaves). In turn, this free-energy fully determines the distribution of large-scale loops in the random graph. We stress that the whole complexity of this cavity procedure is actually hidden in the assumption of self-consistency for the field(s) 20. Assumptions corresponding to broken symmetry in the replica framework [21, 22] may be necessary for models with random interactions. 
Anyhow, the salient feature of the above standard approach is that it predicts global thermodynamical quantities from local considerations about the graph only.

We have added to our exact computation, valid in the $N \longrightarrow \infty$ limit, results from exact enumeration at finite $N$. Thanks to them we have been able to determine precisely the behavior of the leading corrections to the thermodynamical behavior (at least for circuits with $\ell<1$ : we have found that Hamiltonian circuits have stronger finite size corrections and a peculiar finite $N$ behavior.

It would be very interesting to characterize properties or quantities that can adequately be described by a local procedure [23. The question is however well beyond the scope of the present paper, which only shows that large loops are (remarkably) among those ones.

\section{Acknowledgments}

We are grateful to G. Parisi for insights about the validity of replica symmetry for the Ising model with complex temperature. We also thank S. Cocco, A. Montanari for interesting discussions. R.M. thanks the Les Houches summer school and the SMCINFM Center in Rome for the hospitality in August and September 2003 respectively. E.M. thanks the "Laboratoire de Physique Théorique" of ENS in Paris for a visiting professorship during May-June 2004.

\section{References}

[1] S.N. Dorogovtsev, J.F.F. Mendes, Evolution of Networks: From Biological Nets to the Internet and $W W W$ (Oxford University Press, Oxford, UK, 2003)

[2] S. Janson, T. Luczak, A. Rucinski, Random Graphs (John Wiley and Sons, New York, USA, 2000)

[3] M.E.J. Newmann, S.H. Strogatz, D.J. Watts, Phys. Rev. E 64, 026118 (2001).

[4] N.C. Wormald Models of Random Regular Graphs in Surveys in Combinatorics, edited by J.D. Lamb and D.A. Preece, London Mathematical Society Lecture Note Series 276 pp. 239-298 (Cambridge University Press, Cambridge, UK, 1999)

[5] A. Rucinski, N.C. Wormald, Combin. Probab. Comput. 1, 169 (1992)

[6] E. Ben-Naim, P.L. Krapivsky, preprint cond-mat/0403453

[7] H.D. Rozenfeld, J.E. Kirk, E.M. Bollt, D. ben-Avraham, preprint cond-mat/0403536

[8] C. de Dominicis, Y. Goldschmidt, J. Phys. A 22, L775 (1989)

[9] P. Mottishaw, Europhys. Lett. 4, 333 (1987)

[10] I. Kanter, H. Sompolinsky, Phys. Rev. Lett. 58, 164 (1987)

[11] D.R. Bowman, K. Levin, Phys. Rev. B 25, 3438 (1982)

[12] C. Bachas, C. de Calan, P.M.S. Petropoulos, J. Phys. A 27, 6121 (1994)

[13] A. Lefevre, D.S. Dean, Eur. Phys. J. B 21, 121 (2001)

[14] G. Parisi, Statistical Field Theory (Addison Wesley, Redwood City, USA, 1988)

[15] G. Parisi, private communication.

[16] P.G. de Gennes Phys. Lett. 38 A, 339 (1972)

[17] A. Montanari, M. Müller, M. Mezard, Phys. Rev. Lett. 92, 185509 (2004).

[18] H. Garmo, Random Structures Algorithms 15, 43 (1999)

[19] D.B. Johnson, SIAM J. Comput. 4, 77 (1975) 
[20] D.J. Thouless, Phys. Rev. Lett. 56, 1082 (1986)

[21] R. Monasson, J. Phys. A 31, 515 (1998)

[22] M. Mézard, G. Parisi, Eur. Phys. J. B 20, 217 (2001)

[23] D. Aldous, A. Bandyopadhyay, preprint (2004). 